\title{
Distributed Control of Residential Energy Systems using a Market Maker ${ }^{\star}$
}

\author{
Karl Worthmann* Christopher M. Kellett ${ }^{* *}$ Lars Grüne ${ }^{* * *}$ \\ Steven R. Weller ${ }^{* *}$ \\ * Mathematisches Institut, Technische Universität Ilmenau, 99693 \\ Ilmenau, Germany, (e-mail: karl.worthmann@tu-ilmenau.de) \\ ** School of Electrical Engineering and Computer Science, University \\ of Newcastle, Callaghan, NSW 2308, Australia (email: \{chris.kellett, \\ steven.weller $\}$ @newcastle.edu.au) \\ *** Mathematical Institute, University of Bayreuth, Germany (e-mail: \\ lars.gruene@uni-bayreuth.de)
}

\begin{abstract}
The recent rapid uptake of residential solar photovoltaic (PV) installations provides many challenges for electricity distribution networks designed for one-way power flow from the distribution company to the residential customer. In particular, for grid-connected installations, intermittent generation as well as large amounts of generation during low load periods can lead to a degradation of power quality and even outages due to overvoltage conditions. In this paper we present two approaches to mitigate these difficulties using small-scale distributed battery storage. The first is a decentralized model predictive control (MPC) approach while the second is a hierarchical distributed MPC approach using a so-called Market Maker. These approaches are validated and compared using data on load and generation profiles from customers in an Australian electricity distribution network.
\end{abstract}

\section{INTRODUCTION}

Recent years have seen dramatic worldwide growth in residential solar photovoltaic (PV) generation. In Australia, for example, the National Electricity Market (NEM) has seen estimated installed capacity rise from just $23 \mathrm{MW}$ in 2008 to $1450 \mathrm{MW}$ in 2012 with a forecast of $5100 \mathrm{MW}$ by 2020 (Australian Energy Market Operator (AEMO) [2012]). The key drivers for this rapid uptake include the introduction of residential feed-in tariffs, together with sharp falls in the capital costs of solar PV panels.

High levels of PV penetration into the low-voltage distribution network can create significant operational problems for utilities, such as reverse power flow during daytime periods of peak generation coupled with low residential load (Katiraei and Aguero [2011], Huq et al. [2012]). This can in turn lead to increases in distribution feeder voltages (the so-called voltage rise problem), with the potential for adverse impacts on the operation and safety of customerowned devices (Huq et al. [2012]). The intermittency of solar PV generation further exacerbates these problems.

In response to these challenges in integrating solar PV into the grid, the opportunities offered by distributed battery storage devices are increasingly being recognized by utilities looking to reinforce distribution networks and shave peak demand without large-scale capital costs for feeder replacement and related network upgrades (Nykamp et al.

\footnotetext{
* K. Worthmann is supported by the DFG Grant GR1569/12-2. C.M. Kellett is supported by ARC Future Fellowship FT1101000746. L. Grüne is supported by the European Union under the 7 th Framework Programme FP7-PEOPLE-2010-ITN Grant agreement number 264735-SADCO.
}

[2013a], Nykamp et al. [2013b]). Likewise, consumers seeking reduced electricity costs by shifting electricity purchases away from times of peak tariffs, together with a desire for increased energy self-sufficiency, are beginning to consider residential battery storage as a viable option.

To date the high capital cost of battery systems has made deployment of residential energy storage systems largely uneconomic (Wolfs and Reddy [2012]). This situation is set to change in the near future, however, through a confluence of steadily rising electricity costs and projections of rapid uptake of battery electric vehicles (BEVs) and plug-in hybrid electric vehicles (PHEVs) over the next 5-10 years. If realized, these projections point to opportunities for employing retired electric vehicle batteries in residential storage applications, over and above the storage offered by in-service BEVs and PHEVs (Shao et al. [2013], Wolfs and Reddy [2012]).

With economically viable residential storage on the horizon, researchers have recently moved from the analysis of relatively rudimentary and largely uncoordinated battery energy storage systems (Nair and Garimella [2010]) to systems of increasing scale and sophistication (Wolfs and Reddy [2012], Hill et al. [2012], Huq et al. [2012], Tran and Khambadkone [2013], Guo et al. [2013]). A key signature of these more recently proposed systems is their decentralized nature, with the consequent need for distributed control to achieve desired network behavior.

In this paper, we propose a hierarchical distributed control structure for residential energy systems based on the socalled Market Maker concept (Garman [1976]; see also Beja and Goldman [1980]). The basic module is the resi- 
dential energy system (RES) shown in Figure 1, consisting of the residential load, a battery, and solar PV panels. Each RES is connected to the wider electricity network through a point of common coupling.

Let $\mathcal{I}$ be the number of RESs in the network, and assume the existence of a Market Maker (MM) or Local Grid Operator (we will use Market Maker in what follows). We assume that each RES can communicate with the Market Maker, but that the RESs do not communicate with each other, reflecting the largely ad hoc formation of residential energy systems. Each RES implements a model-predictive control (MPC) strategy (Grüne and Pannek [2011]) in order to determine its grid (voltage) profile by modulating its battery profile. The Market Maker implements a simple strategy in order to set prices for buying and selling electricity within the residential network, with a view to minimizing the aggregate impact of the $\mathcal{I}$ RESs on the distribution grid.

In Ratnam et al. [2013], a minimization criterion was introduced in order to reduce the impact of a single RES on the power grid. To achieve this goal, drawing and supplying power from/to the grid were equally penalized. While the present paper starts from the RES setup proposed in Ratnam et al. [2013], we propose three important extensions. The first is that we consider an interconnected network of several RESs as opposed to a single RES. The second is that we aim to maximize the profit of (or minimize the cost to) each individual RES. The third modification is that we seek in the present paper to maximize profit in an ongoing manner by implementing the optimization in a receding horizon (or MPC) fashion.

The rest of the paper is organized as follows: in Section 2 we formalize the Residential Energy System. In Section 3 we present a decentralized MPC scheme for the network (i.e., a scheme without communication or cooperation among RESs). In Section 4 we formally introduce the Market Maker and propose a distributed MPC scheme using the Market Maker. In Section 5 we apply these algorithms to data from an Australian electricity distribution company to investigate the behavior of the proposed algorithms when applied to a real-world setting. We conclude in Section 6 .

In what follows we denote the positive integers by $\mathbb{N}$ and the nonnegative integers by $\mathbb{N}_{0}$.

\section{THE RESIDENTIAL ENERGY SYSTEM}

We consider a small, neighborhood-level, electricity network consisting of several residences. Each residence comprises a Residential Energy System (RES) as shown in Figure 1, consisting of a residential load, a battery, and solar PV panels. Each RES is connected to the wider electricity network. In what follows, the solar PV panels could be replaced by any residential-scale local generation and the battery could be replaced by any residentialscale local energy storage. The important characteristics of these elements are that the generation and the load are not controllable. A recent study (Barker et al. [2012]) suggests that flexible consumption represents up to $60 \%$ of household electricity usage in the form of appliances such as air conditioners and refrigerators. Consequently, in

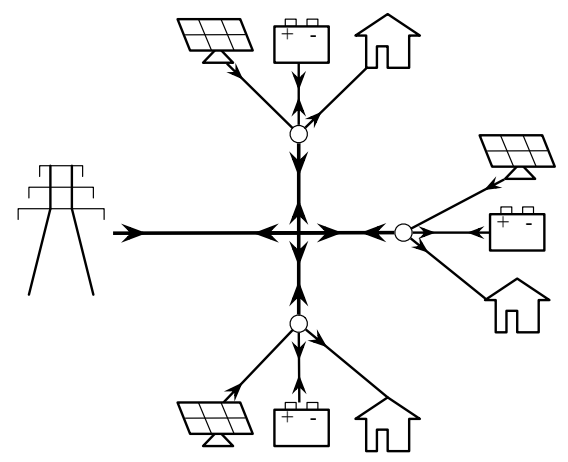

Fig. 1. System model where possible power transfer is indicated by an arrow above. In particular, solar panels provide power, residential loads draw power, and the batteries and network can both supply and draw power.

future work we will include splitting the load into controllable and uncontrollable components but, for simplicity, we initially restrict attention to uncontrollable loads only.

Mathematically, the RES is defined by the following discrete-time system

$$
\begin{aligned}
x(k+1) & =f(x(k), u(k)), \\
y(k) & =h(u(k), w(k))
\end{aligned}
$$

where $x, u, w \in \mathbb{R}^{\mathcal{I}}$ and $\mathcal{I} \in \mathbb{N}$ is the number of RESs connected in the local area under consideration. For user $i, x_{i}$ is the state of charge of the battery in $\mathrm{kWh}, u_{i}$ is the battery charge/discharge rate in $\mathrm{kW}, w_{i}$ is the residential load minus the local generation in $\mathrm{kW}$, and $y_{i}$ is the power supplied by/to the grid in $\mathrm{kW}$. A simple model of the RES of user $i$ is:

$$
\begin{aligned}
x_{i}(k+1) & =x_{i}(k)+T u_{i}(k), \\
y_{i}(k) & =w_{i}(k)+u_{i}(k) .
\end{aligned}
$$

Here, $T$ represents the length of the sampling interval in hours; e.g., $T=0.5$ corresponds to 30 minutes. The state of charge of the battery and the charge/discharge rates of the battery are constrained in practice. In other words, there exist $C_{i}, \bar{u}_{i} \in \mathbb{R}_{>0}$ and $\underline{u}_{i} \in \mathbb{R}_{<0}$ so that

$$
0 \leq x_{i}(k) \leq \bar{C}_{i} \quad \forall k \in \mathbb{N}_{0}
$$

and

$$
\underline{u}_{i} \leq u_{i}(k) \leq \bar{u}_{i} \quad \forall k \in \mathbb{N}_{0}
$$

A simple approach to using a battery in an RES, which we refer to as the Simple Controller, is as follows: If generation exceeds load, and if the battery is not fully charged, then charge the battery. If load exceeds generation, and if the battery is not fully discharged, then discharge the battery (see, e.g., Nykamp et al. [2013b]). In a scenario where power can be sold by a residence to the grid, such as is considered herein, this behavior can be enforced by setting the price for buying power (slightly) higher than the price for selling power. We assume that metering is in place to allow each RES to purchase electricity from the grid as well as to receive compensation for electricity delivered to the grid. This is common practice in electricity networks where there is a feed-in tariff for power supplied to the grid by individual residences.

To facilitate our discussion of algorithms for decentralized or distributed control of RESs we will make use of the three synthetic energy profiles $w_{i}$ depicted in Figure 2. 


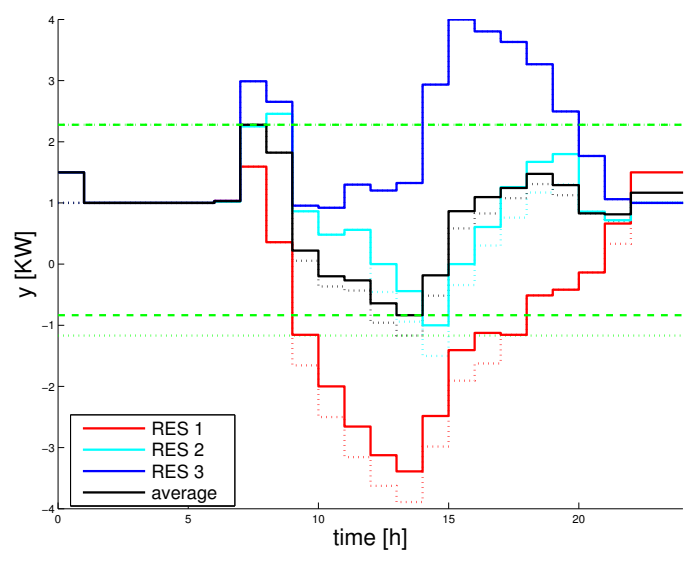

Fig. 2. Energy usage without (...) and with (-) use of battery storage is shown for 24 hours. Peaks are indicated by green lines.

\section{A DECENTRALIZED RES CONTROLLER}

For the Simple Controller proposed in the previous section, if the load always exceeds generation then the battery will never be used (see RES 3 in Figure 2). Intuitively, though, a battery can be used to time-shift energy consumption and, hence, can be used to flatten the usage profile. Consequently, in this section we present a simple decentralized MPC scheme such that each RES uses a local controller in order to even out its profile, $\left(y_{i}(k)\right)_{k \in \mathbb{N}_{0}}, i \in\{1,2, \ldots, \mathcal{I}\}$, of power supplied from/to the grid.

MPC is a control strategy that aims at improving system behavior by iteratively minimizing an optimization criterion with respect to predicted trajectories and implementing the first part of the resulting optimal control sequence until the next optimization is performed, see Grüne and Pannek [2011] for details. We propose such a predictive controller for (3). In order to do this, we assume that we have predictions of the residential load and generation some time into the future that is coincident with the horizon of the predictive controller. In other words, given a prediction horizon $N \in \mathbb{N}$, we assume knowledge of $w_{i}(j)$ for all $j \in\{k, \ldots, k+N-1\}$, where $k \in \mathbb{N}_{0}$ is the current time. If the prediction horizon is less than a day, i.e. $N T \leq 24$, such an assumption is not initially unreasonable as residential loads tend to follow daily patterns and one-day ahead weather predictions can be fairly accurate. Future work will involve investigating sensitivity of the proposed scheme to the accuracy of these predictions as well as to the prediction horizon length.

A straightforward option in order to flatten the energy profile of the $i$-th RES is to penalize deviations from its (anticipated) average "consumption", defined as

$$
\zeta_{i}(k):=\frac{1}{N} \sum_{j=0}^{N-1} w_{i}(k+j) .
$$

With a quadratic cost function, this leads to the finitehorizon optimal control problem

$$
\min _{\hat{u}_{i}(\cdot)} \sum_{j=0}^{N-1}\left(\hat{y}_{i}(k+j)-\zeta_{i}(k)\right)^{2}
$$

subject to the system dynamics (3), the current state, and the battery constraints (4)-(5). Throughout this paper we set $x(0)=0.5, C_{i}=4, \underline{u}_{i}=-0.5$, and $\bar{u}_{i}=0.5$. In comparison with the Simple Controller of the previous section, this decentralized control algorithm reduces the maximum aggregate demand to 1.7785 (from 2.2785) and reduces the maximum aggregate supply from the RESs to 0.8259 (from 1.1683), see also Table 1 in Section 4. In particular, the peak energy consumption is reduced by 0.5 which corresponds to the maximal charging rate, see also Figure 3 on the left.
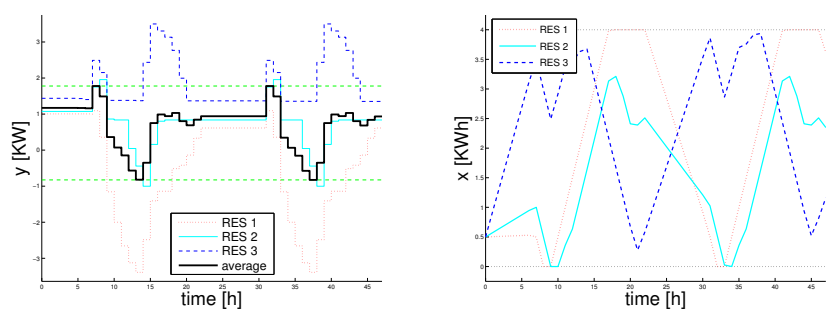

Fig. 3. Decentralized MPC with one day prediction horizon: output (left) and state (right) trajectories $y_{i}(k)$ and $x_{i}(k), k=0,1, \ldots, 47$, respectively.

Furthermore, a look at the corresponding state trajectories on the right in Figure 3 reveals that all subsystems make use of their batteries in order to flatten their individual energy profiles. In particular, we note that using the Simple Controller of the previous section, RES 3 did not use its battery at all, whereas using the decentralized MPC scheme RES 3 always discharges its battery during its period of peak demand.

\section{A DISTRIBUTED APPROACH}

In the decentralized approach of the previous section there is no coordination between RESs. In this section, we propose a hierarchical distributed control approach where each RES can communicate with a centralized entity, called the Market Maker (MM) (Garman [1976]), with the aim of achieving some network-wide objective. The objective we pursue in this work is to flatten the aggregate power usage of the network.

In what follows the price of buying or selling power from or to the grid, respectively, for an RES is discussed. It is important in this context to note that these need not be monetary prices, but, rather, can be viewed as a mechanism to enforce reasonable cooperation between RESs within the network. However, in applications such as isolated or islanded microgrids, the distributed control approach we propose herein may indeed form the basis of an electricity market.

Denote the price for buying power from the grid by $p$ : $\mathbb{N}_{0} \rightarrow \mathbb{R}_{>0}$ and the price to sell power to the grid by $q: \mathbb{N}_{0} \rightarrow \mathbb{R}_{>0}$. In many current electricity markets the values of $p$ and $q$ are constant; i.e., they do not depend on the time index $k$. In markets that implement time-ofuse pricing, the $p$ and $q$ are periodic with a period of 24 hours and with higher values at times of predicted high usage and lower values at times of predicted low usage. In the sequel, these prices will be manipulated by the Market 
Maker in real-time in order to obtain desirable behavior from the residential network. To this end, the quantities

$$
\begin{aligned}
& y^{+}(k) \doteq \max \{y(k), 0\}, \quad \text { and } \\
& y^{-}(k) \doteq \max \{-y(k), 0\} .
\end{aligned}
$$

are defined. We observe that only one of $y^{+}(k)$ or $y^{-}(k)$ can be nonzero at each $k$. We also observe that $y^{+}(k)$ is the power drawn from the grid at time $k$ while $y^{-}(k)$ is the power supplied to the grid at time $k$. We denote the $N \times N$ identity matrix by $I_{N}$, the $N$ vector $(1,1, \ldots, 1)^{T}$ by $\mathbb{1}_{N}$, the $N \times N$ matrix of all zeros by $\mathbf{0}_{N}$, and the $N \times N$ lower triangular matrix consisting of ones and zeros by

$$
L_{N}=\left[\begin{array}{ccc}
1 & & 0 \\
\vdots & \ddots & \\
1 & \cdots & 1
\end{array}\right]
$$

We define the finite-horizon optimal control problem for each RES so as to minimize the cost of an individual residence over an $N$-step horizon; i.e.,

$$
\min _{\hat{u}_{i}(\cdot)} \underbrace{\sum_{j=k}^{k+N-1} p(j) \hat{y}_{i}^{+}(j)-q(j) \hat{y}_{i}^{-}(j)}_{=0^{T} \hat{u}+p^{T} \hat{y}^{+}-q^{T} \hat{y}^{-}}
$$

subject to the constraints (4) representing the battery capacities and system dynamics $(3)$; i.e., defining $\widetilde{w}_{i}(k) \in$ $\mathbb{R}^{N}$ by $\widetilde{w}_{i}(k):=\left[w_{i}(k), w_{i}(k+1), \ldots, w_{i}(k+N-1)\right]^{T}$ we have the constraints

$$
\left[\begin{array}{rrr}
T \cdot L_{N} & \mathbf{0}_{N} & \mathbf{0}_{N} \\
-T \cdot L_{N} & \mathbf{0}_{N} & \mathbf{0}_{N} \\
-I_{N} & I_{N} & -I_{N} \\
I_{N} & -I_{N} & I_{N}
\end{array}\right]\left(\begin{array}{l}
\hat{u}_{i} \\
\hat{y}_{i}^{+} \\
\hat{y}_{i}^{-}
\end{array}\right) \leq\left(\frac{\frac{\left(C-x_{i}(k)\right) \cdot \mathbb{1}_{N}}{x_{i}(k) \cdot \mathbb{1}_{N}}}{\frac{\widetilde{w}_{i}(k)}{-\widetilde{w}_{i}(k)}}\right)
$$

as well as the constraints (5); i.e., $\underline{u} \cdot \mathbb{1}_{N} \leq \hat{u}_{i} \leq \bar{u} \cdot \mathbb{1}_{N}$. Here, the optimization variables are

$$
\begin{aligned}
\hat{u}_{i} & =\left(\hat{u}_{i}(k), \ldots, \hat{u}_{i}(k+N-1)\right)^{T}, \\
\hat{y}_{i}^{+} & =\left(\hat{y}_{i}^{+}(k), \ldots, \hat{y}_{i}^{+}(k+N-1)\right)^{T}, \\
\hat{y}_{i}^{-} & =\left(\hat{y}_{i}^{-}(k), \ldots, \hat{y}_{i}^{-}(k+N-1)\right)^{T} .
\end{aligned}
$$

Since this is a linear optimization problem, the optimum is attained in each minimization - although it may not be unique. Note that the minimization problem to be solved encompasses $3 N$ variables and $6 N$ constraints yielding linear growth of the optimization variables and constraints in the prediction horizon. Furthermore, it can be observed that any predicted control yields a solution that is necessarily zero at the end of the prediction horizon. This is intuitively obvious since there is no benefit to having a charge left in the battery at the end of the horizon. However, this does not necessarily imply that, for a fixed $k, x_{i}(k+N-1)$ is necessarily zero due to the receding horizon nature of MPC.

\subsection{The Market Maker}

In order to set prices, we propose an iterative negotiation before prices are set. This negotiation is operated by the Market Maker, who sets initial prices from the current time $k$ to the end of the prediction horizon $k+N-1$ and broadcasts these to the residential network. Each RES will then solve its own MPC problem based on the cost functional given by (6) and then communicate its desired grid profile, $\left\{\hat{y}_{i}(j)\right\}_{j=k}^{k+N-1}$, to the Market Maker. The Market Maker uses the aggregated grid profile to update prices, which are then broadcast to the residential network. This process is iterated until (hopefully) a steadystate is reached. Note that in a slight modification to the original definition of a Market Maker proposed in Garman [1976] we allow multiple iterations of the Market Maker setting prices and receiving bids whereas in Garman [1976] the Market Maker receives bids once and sets prices once (i.e., there is only a single iteration per sampling instant). Herein we propose a simple algorithm for the setting of prices by the Market Maker. However many algorithms are possible (see, e.g., Slamka et al. [2013]) and investigating these alternatives is the subject of ongoing work.

Denote the negotiation iteration index by $\ell \in \mathbb{N}_{0}$ and the predicted grid profile for $\operatorname{RES} i, i \in\{1, \ldots, \mathcal{I}\}$, at negotiation iteration $\ell$ by $\left\{\hat{y}_{i, \ell}(j)\right\}_{j=k}^{k+N-1}$. Denote the predicted demand of the residential network at time $j$ and negotiation iteration $\ell$ by

$$
\Pi_{\ell}(j) \doteq \frac{1}{\mathcal{I}} \sum_{i=1}^{\mathcal{I}} \hat{y}_{i, \ell}(j) \quad \forall j \in\{k, \ldots, k+N-1\}
$$

and the average predicted demand as

$$
\bar{\Pi}(j) \doteq \frac{1}{N} \sum_{j=k}^{k+N-1} \Pi_{\ell}(j) .
$$

Note that since the predicted residential load and generation is fixed for the prediction horizon, the average predicted demand, $\bar{\Pi}(j)$, is independent of the iterative negotiation process.

Let $\underline{p}, \bar{p} \in \mathbb{R}_{\geq 0}$ be the minimum and maximum buying prices, respectively. The buying price $p_{\ell+1}(j)$ of the successor iteration is set by the Market Maker as

$$
p_{\ell+1}(j)=\max \left\{\underline{p}, \min \left\{\bar{p}, p_{\ell}(j)+\theta\left(\Pi_{\ell}(j)-\bar{\Pi}(j)\right)\right\}\right\}
$$

where $\theta \in \mathbb{R}_{>0}$ is a selectable parameter. The selling price $q_{\ell+1}(j)$ is set to a fraction of $p_{\ell+1}(j)$; i.e. $q_{\ell+1}(j)=$ $\kappa p_{\ell+1}(j), \kappa \in(0,1)$. This convention ensures that certain pathological arbitrage-type behavior is avoided since $p_{\ell}(j) \geq q_{\ell}(j)$ for all $\ell$ and $j$. In other words, buying electricity at a particular time is always more expensive than what can be obtained by selling electricity at that time. This inhibits an RES short-selling electricity; i.e., buying a lot of power to charge its battery at one time instant and then turning around and selling that power at the next time instant at a profit. Note that in the presence of realistic charging/discharging rate constraints this may not be a problem. In the following simulations we set $\theta=0.05, \kappa=0.95, p=0, \bar{p}=10$, and we perform three Market Maker iterations; i.e., $\ell=0,1,2,3$.

We see that when network demand at time $j$ exceeds the average predicted demand, the Market Maker increases both the selling and buying price at time $k$ and, conversely, if the network demand is less than the average predicted demand the Market Maker decreases the prices. Intuitively, this should have the effect of flattening the aggregate power drawn or supplied from the residential energy network, cf. Figure 4. 


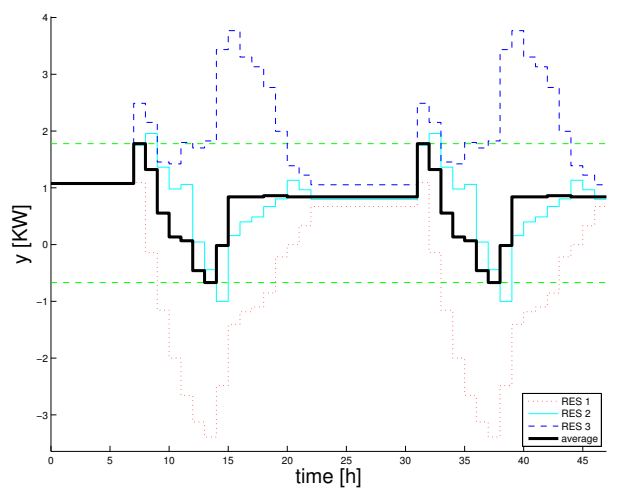

Fig. 4. Distributed MPC with one day prediction horizon: output trajectories $y_{i}(k), k=0,1, \ldots, 47$.

\begin{tabular}{|r|c|c|c|}
\hline & peak (high) & peak (low) & variation \\
\hline No Battery Storage & 2.2785 & -1.1683 & 3.4468 \\
\hline Simple Controller & 2.2785 & -0.8350 & 3.1135 \\
\hline Decentralized MPC & 1.7785 & -0.8259 & 2.6044 \\
\hline Distributed MPC & 1.7785 & -0.6683 & 2.4468 \\
\hline
\end{tabular}

Table 1. Highest and lowest energy demand over the simulation time horizon and the difference between those two values for different control techniques (synthetic profiles).

For the load/generation profiles presented in Figure 2, the maximal achievable reduction with respect to the peaks is attained and the decentralized MPC algorithm is outperformed, cf. Table 1. In order to explain this performance improvement, the corresponding charge levels of the batteries are considered, see Figure 5 .

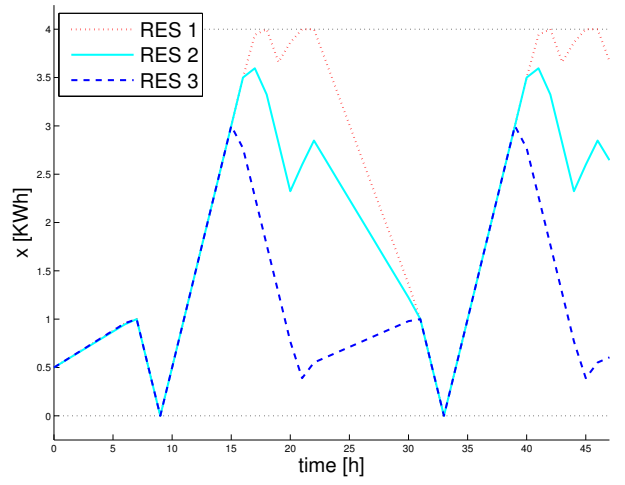

Fig. 5. State trajectories resulting from distributed MPC, i.e. battery levels of the individual subsystems.

In particular, it is remarkable that the decisions of individual subsystems are more 'in line'. In the decentralized MPC setting, subsystem RES 3 discharged its battery after noon (14-15 hours) but now charges during this period of time since the overall energy demand is far below the average, as can be seen in Figure 4.

Remark 1. The storage capacity of the individual subsystems does not have to be equal. This restriction was employed in order to simplify the presentation of our numerical findings.

\begin{tabular}{|r|c|c|c|}
\hline & peak (high) & peak (low) & variation \\
\hline No Battery Storage & 1.0540 & -0.4454 & 1.4994 \\
\hline Simple Controller & 1.1040 & -0.2360 & 1.3400 \\
\hline Decentralized MPC & 0.8747 & -0.0308 & 0.9055 \\
\hline Distributed MPC & 0.7209 & -0.0329 & 0.7538 \\
\hline
\end{tabular}

Table 2. Energy demand peak values for different control techniques (Ausgrid data).

\section{AUSTRALIAN DATA}

In this section, we compare the three previously discussed controllers by considering the load and generation profiles for 10 customers drawn from the Australian electricity distribution company Ausgrid, a state-owned corporation servicing approximately 1.6 million customers across New South Wales from Sydney to Newcastle. The data from these customers was collected as part of the Smart Grid, Smart City project and covers the period 1-7 March 2011.

The difference of load and generation is shown in Figure 6. Here, the mean consumption is +0.2308 while the peaks of the depicted average trajectory are +1.0540 (high) and -0.4454 (low). Note that this is the no battery case.

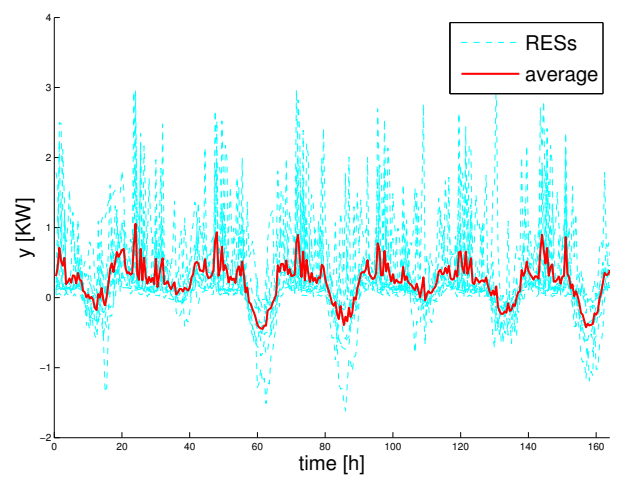

Fig. 6. Energy drawn from / supplied to the grid for ten RESs during the first week of March 2011.

Applying the decentralized MPC scheme of Section 3 yields the load/generation and battery profiles shown in Figure 7. Applying the Market Maker based distributed MPC scheme of Section 4 yields the load/generation and battery profiles shown in Figure 8 . The peak values are shown in Table 2. We observe that for a network composed solely of these customers, the peak power demand and the peak power the network needs to absorb are both significantly reduced by both MPC schemes, with the Market Maker distributed MPC scheme outperforming the decentralized MPC scheme.

We note that the distributed MPC scheme appears to better utilize the available battery storage when compared with the decentralized scheme in the sense that for the decentralized scheme the batteries are on average never empty. In contrast to this, in the distributed setting their capacity is completely employed in order to further flatten the aggregate grid profile. 

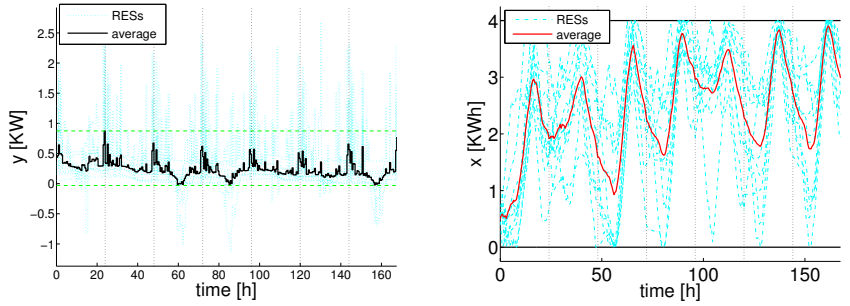

Fig. 7. Decentralized MPC applied to RESs depicted in Figure 6: Energy drawn from / supplied to the grid (left) and battery state of charge (right).
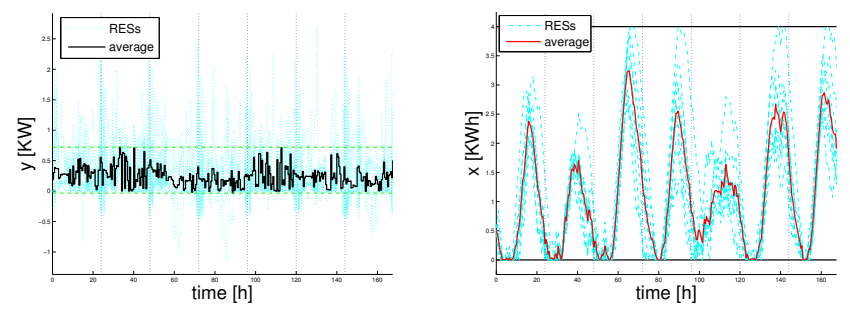

Fig. 8. Distributed MPC applied for RESs depicted in Figure 6: Energy drawn from / supplied to the grid (left) and battery state of charge (right).

\section{CONCLUSIONS}

Residences with small-scale solar generation, such as roofmounted solar PV, are becoming increasingly common and battery storage is frequently cited as a technology to mitigate some of the negative network impacts that come with widespread uptake of distributed electricity generation. In this paper we have presented decentralized and distributed MPC schemes for control of a network of residences, each of which comprises a residential energy system having a load, local generation, and a local storage element. Our proposed distributed MPC scheme relies on an idea from the economics literature: the Market Maker (Garman [1976]). The effectiveness of both the decentralized and distributed MPC schemes was demonstrated on data from customers in New South Wales, Australia.

Both the model and approach taken herein are intentionally simple, in order to verify the soundness of the broad algorithmic approach. There are, of course, many complications to be overcome to move the proposed distributed MPC implementation into practice. A non-exhaustive list includes: investigating the sensitivity of the algorithm to imperfect prediction of load and generation; investigating the communication requirements of the algorithm, in particular the sensitivity to communicating only coarsely quantized trajectories to the Market Maker and coarsely quantized prices to the customers; and making use of more realistic battery models in which preferred operating modes of different battery types are taken into account (Tran and Khambadkone [2013]). Furthermore, investigating different algorithms for the operation of the Market Maker may lead to improved performance.

\section{ACKNOWLEDGEMENTS}

The assistance of E. Ratnam in facilitating access to the Ausgrid data is gratefully acknowledged.

\section{REFERENCES}

Australian Energy Market Operator (AEMO). Rooftop PV information paper (National Electricity Forecasting), May 2012.

S. Barker, A. Mishra, D. Irwin, P. Shenoy, and J. Albrecht. SmartCap: Flattening peak electricity demand in smart homes. In Proc. IEEE Int. Conf. on Pervasive Computing and Communications, Lugano, Switzerland, 2012.

A. Beja and M. B. Goldman. On the dynamic behavior of prices in disequilibrium. J. Financ., 35(2):235-248, 1980.

M. B. Garman. Market microstructure. J. Financ. Econ., 3:257-275, 1976.

L. Grüne and J. Pannek. Nonlinear Model Predictive Control. Theory and Algorithms. Springer London, 2011.

Y. Guo, M. Pan, Y. Fang, and P. P. Khargonekar. Decentralized coordination of energy utilization for residential households in the smart grid. IEEE Trans. Smart Grid, 4(3):1341-1350, 2013.

C. A. Hill, M. C. Such, D. Chen, J. Gonzalez, and W. M. Grady. Battery energy storage for enabling integration of distributed solar power generation. IEEE Trans. Smart Grid, 3(2):850-857, 2012.

K. M. M. Huq, M. E. Baran, S. Lukic, and O. E. Nare. An energy management system for a community energy storage system. In Proc. IEEE Energy Conversion Congress and Exposition, 2012.

F. Katiraei and J. R. Aguero. Solar PV integration challenges. IEEE Power Energy Mag., 9(3):62-71, 2011.

N.-K. C. Nair and N. Garimella. Battery energy storage systems: Assessment for small-scale renewable energy integration. Energ. Buildings, 42(11):2124-2130, 2010.

S. Nykamp, M. G. C. Bosman, A. Molderink, J. L. Hurink, and G. J. M. Smit. Value of storage in distribution grids - Competition or cooperation of stakeholders? IEEE Trans. Smart Grid, 4(3):1361-1370, 2013a.

S. Nykamp, A. Molderink, J. L. Hurink, and G. J. M. Smit. Storage operation for peak shaving of distributed $\mathrm{PV}$ and wind generation. In Proc. IEEE PES Innovative Smart Grid Technologies, 2013b.

E. L. Ratnam, S. R. Weller, and C. M. Kellett. An optimization-based approach for assessing the benefits of residential battery storage in conjunction with solar PV. In Proc. 2013 IREP Symp. Bulk Power System Dynamics and Control-IX, Rethymnon, Greece, 2013.

S. Shao, F. Jahanbakhsh, J. R. Agüero, and L. Xu. Integration of PEVs and PV-DG in power distribution systems using distributed energy storage - Dynamic analyses. In Proc. IEEE PES Innovative Smart Grid Technologies (IGST), 2013.

C. Slamka, B. Skiera, and M. Spann. Prediction market performance and market liquidity: A comparison of automated market makers. IEEE Trans. Eng. Manag., 60(1):169-185, 2013.

D. Tran and A. M. Khambadkone. Energy management for lifetime extension of energy storage system in micro-grid applications. IEEE Trans. Smart Grid, 4(3):1289-1296, 2013.

P. Wolfs and G. S. Reddy. A receding predictive horizon approach to the periodic optimization of community battery energy storage systems. In Proc. 22nd Australasian Universities Power Engineering Conf., 2012. 